\title{
Agreement of young adults and orthodontists on dental aesthetics \& influencing factors of self-perceived aesthetics
}

Ying Cai ${ }^{1}$, Wulong Du², Feiou Lin ${ }^{1,3^{*}}$, Shengjia Ye ${ }^{2}$ and Yanling Ye ${ }^{2}$

\begin{abstract}
Background: The aim of this study is to assess the agreement between orthodontist's and Chinese young adult's self-perceived aesthetics, the normative treatment need based on the Index of Orthodontic Treatment Need (IOTN), and the main factors affecting the self-perceived aesthetics.

Methods: A random sample of 348 Chinese young adults (116 males and 232 females) aged 17-24 years were recruited in this study. Two orthodontists were involved in rating the cases. Orthodontic treatment need was assessed according to the Index of Orthodontic Treatment Need, including Aesthetic components and Dental Health Components (AC and DHC). Personality traits were assessed according to Eysenck Personality Questionnaire (EPQ). Cohen's kappa test was used to assess the agreement, and spearman's correlation coefficient was used to analyze the association among all variables.

Results: A statistically significant level of agreement was observed between young adult's perception and orthodontist's perception in IOTN (kappa $=0.14)$. A positive relationship $(p<0.001)$ existed between the young adult's AC and the orthodontist' s AC ( $r=0.275)$, and between the young adult's AC and the normative need DHC $(r=0.195)$. The orthodontist's AC was strongly related to the normative need $(r=0.743, p<0.001)$. Association between the young adult's AC and gender and EPQ-E were also observed.

Conclusions: Young adults tend to be less critical in assessing orthodontic treatment needs than orthodontists. The orthodontist's AC reflecting subjective treatment need is strongly connected to the normative need. The adult's perception of aesthetic component is affected by factors such as gender and personality traits.
\end{abstract}

Keywords: IOTN, Aesthetic component, Personality, Agreement

\section{Background}

The subjective orthodontic treatment need of Chinese natives is high [1]. Apart from the dental health needs, it is necessary to take subjective aesthetic into consideration as well [2]. Among the Chinese young adults, the common motivation for orthodontic treatment is associated with the desire for improvement in appearance [3].

\footnotetext{
*Correspondence: linfeiousci@163.com

'Department of Orthodontics, School and Hospital of Stomatology, Wenzhou Medical University, West Xueyuan Road 373, Wenzhou, Zhejiang 325000, People's Republic of China

${ }^{3}$ School and Hospital of Stomatology, Shandong University, Jinan, Shandong, People's Republic of China

Full list of author information is available at the end of the article
}

An attractive facial appearance has a positive impact on socio-psychological well-being as well as professional relations [4]. It is also suggested that dental esthetics may be important in individual oral-health attitudes and behaviors [5-7]. When young adults are seeking orthodontic treatment, it is appropriate for orthodontists to consider the patient's aesthetic component, since this component may influence the communication between the patient and the orthodontist, which will in turn enable the orthodontist to assess his or her expectations. The self-perceptions of young adults are important indicators when deciding to undertake treatment, and this might complement

(c) The Author(s). 2018 Open Access This article is distributed under the terms of the Creative Commons Attribution 4.0 International License (http://creativecommons.org/licenses/by/4.0/), which permits unrestricted use, distribution, and reproduction in any medium, provided you give appropriate credit to the original author(s) and the source, provide a link to the Creative Commons license, and indicate if changes were made. The Creative Commons Public Domain Dedication waiver (http://creativecommons.org/publicdomain/zero/1.0/) applies to the data made available in this article, unless otherwise stated. 
conventional clinical evaluation. However in practice, the patient's own perception is often ignored by most orthodontic treatment need indices [8].

The perceptions of esthetics maybe differ between laypersons and orthodontists. Many studies reveal that the patient's perception is not aligned with the orthodontist's opinion [9-12]. They thought that the professional opinions of orthodontists are generally more critical than those of laypersons when assessing dento-facial esthetics. However, few authors have investigated the self-perceptions of dental esthetics among young adults.

In recently, many orthodontic need questionnaires have been developed and used as outcome measures. The index of orthodontic treatment need (IOTN) consists of the Aesthetic Component (AC) and the Dental Health Component (DHC), being used to rank malocclusion. AC is the subjective part, associated with the assessment of malocclusion [13]. Although, the previous study showed there is only a moderate agreement between $\mathrm{AC}$ and DHC [14].This difference between the DHC and AC reflects that $\mathrm{AC}$ assesses the aesthetic aspects of the malocclusion, only in frontal view, and highlights the subjective nature of it. Therefore, any clinician who is interested in using the IOTN should receive proper training and undergoes the calibration process [15]. However, most researchers found that the IOTN was considered a reproducible and effective way to ascertain the patient's perception of the esthetics [16-20].

Thus, the aim of this study is to assess the agreement between orthodontist's and Chinese young adult's self-perceived aesthetics, the normative treatment need based on the Index of Orthodontic Treatment Need (IOTN), and the main factors affecting the self-perceived aesthetics.

\section{Methods}

All of the protocols were reviewed and approved by the Health Research Ethics Board of Wenzhou Medical University. Written informed consent (including the release for dental records) was obtained from each participant.

\section{Subjects}

It was estimated that a sample size of 48 subjects would be needed to demonstrate a significant change in OHRQoL, with an $80 \%$ probability power at the $5 \%$ level of significance. The sample size was inflated by a $10 \%$ margin to allow for loss to follow-up and dropouts; thus, the total sample size was a minimum of 52 .

A total of 348 young adults (116 males and 232 females) aged $17-24$ years (mean $20.37 \pm 1.22$ years) were randomly selected from two universities in Wenzhou, China from March 2017-May 2017. The distribution of gender
Table 1 Descriptive Statistics by Gender, Age

\begin{tabular}{lllll}
\hline & & $N(\%)$ & Mean & SD \\
\hline \multirow{3}{*}{ gender } & All & 348 & & \\
& Male & $232(66.7)$ & & \\
age & female & $116(33.3)$ & & \\
& 17.00 & $7(2.0)$ & 20.37 & 1.33 \\
& 18.00 & $18(5.2)$ & & \\
& 19.00 & $65(18.7)$ & & \\
& 20.00 & $97(27.9)$ & & \\
& 21.00 & $83(23.9)$ & & \\
& 22.00 & $66(19.0)$ & & \\
& 23.00 & $11(3.2)$ & \\
& 24.00 & $1(0.3)$ & \\
\hline
\end{tabular}

was balanced: males represented $33.3 \%$ and females $66.7 \%$ of the sample population (Table 1). Exclusion criteria included patients with cognitive disorders or chronic medical conditions, those who had previously received any type of orthodontic treatment, and those with craniofacial anomalies such as cleft lip and palate. Participants who did not consent or were undergoing orthodontic treatment were also not included in the study. The data was collected from a clinical inspection by the two orthodontists. All of the questionnaires were filled out within a 30-min time frame and collected on the spot. The questionnaires included the index of orthodontic treatment need (IOTN), Eysenck Personality Questionnaire (EPQ).

\section{Questionnaires IOTN}

About young adults perceived Aesthetic Component (young adult's AC), everyone was shown the 10 photographs of the IOTN and was asked to select the photograph that best represented his/her dental appearance. The Aesthetic Component assessed by orthodontists (orthodontist's AC) of the IOTN and the Dental Health Component (DHC) was recorded by the author. AC ranks attractiveness of dental appearance on a scale of $1-10$, from the least attractive to the most attractive. DHC ranks malocclusions on a scale of 1-5 according to the severity of occlusion traits. AC grades 1 to 4 and DHC grades 1 and 2 represent little or no need for treatment, $\mathrm{AC}$ grades 5 to 7 and $\mathrm{DHC}$ grade 3 a borderline need, and finally AC grades 8 to 10 and DHC grades 4 and 5 a definite need for treatment.

\section{Personality traits}

The Chinese version of EPQ [21, 22], an adaption of the personality traits, was used to assess Chinese respondent. Eighty eight items consisted of yes/no questions were shown to the participants. The whole scale includes four factors, which are extroversion/introversion 
(EPQ-E, representing sociability, liveliness, and positive), Neuroticism (EPQ-N, representing emotional instability and anxiousness), psychoticism (EPQ-P, representing tough-mindedness, aggressiveness, coldness, and egocentricity), and lie (EPQ-L). The EPQ-L is a control scale reflecting the individual level of social naivety [21,23].

\section{Statistical analyses}

Cohen's kappa test was used to assess the consistency of the AC grade determined by young adults and orthodontists, as well as the agreement of orthodontist's AC and DHC [24]. The chi-square test was applied to test distribution differences between the young adult's AC and the orthodontist's AC of the IOTN, and the differences between males and females. Spearman's correlation coefficient was used to identify whether there is a correlation among young adult's AC, orthodontist's AC, DHC, personality traits and gender [25]. The statistical analysis was performed using the Statistical Package for Social Sciences (SPSS, version 19.0 for Windows; Chicago, IL, USA). A $p$ value less than or equal to 0.05 was considered statistically significant $(p \leq 0.05)$.

\section{Results}

A statistically significant level of agreement was observed between young adults and orthodontist perception $($ kappa $=0.14)$. A significant positive relationship existed between the young adult and orthodontist's AC scores $(r=0.275, p<0.001)$, and between young adult's $\mathrm{AC}$ and DHC $(r=0.195, p<0.001)$. The orthodontist's AC was strongly related with the normative need $(r=$ $0.743, p<0.001)$, but only a moderate agreement was observed (kappa $=0.332, p<0.001)$. Correlations between the young adult's AC and gender $(r=0.143, p=0.007)$, EPQ-E were also observed $(r=-0.112, p=0.037)$.

The results of the frequency measured according to young adult's $\mathrm{AC}$, orthodontist's $\mathrm{AC}$ and $\mathrm{DHC}$ of the IOTN are shown in Table 2. Orthodontists perceived $63.8 \%$ to be in mild category with regards to AC (grade 1 to grade 4), which was lower than young adult's self-perception, $30.2 \%$ to be in moderate category (grade 5 to grade 7), 6\% to be in sever category (grade 8 to grade 10), which is higher compared to young adult's

Table 2 Frequency of treatment need according to AC and $\mathrm{DHC}$

\begin{tabular}{llll}
\hline IOTN & DHC $(\mathrm{n} \%)$ & $\begin{array}{l}\text { Young adult's } \\
\mathrm{AC}(\mathrm{n} \%)\end{array}$ & $\begin{array}{l}\text { Orthodontist's } \\
\mathrm{AC}(\mathrm{n} \%)\end{array}$ \\
\hline 1 & $137(39.4)$ & $313(89.9)$ & $222(63.8)$ \\
2 & $127(36.5)$ & $23(6.6)$ & $105(30.2)$ \\
3 & $84(24.1)$ & $12(3.4)$ & $21(6.0)$ \\
Total & 348 & 348 & 348 \\
\hline
\end{tabular}

1 mean: little or no need for treatment; 2 mean: borderline need; 3 mean: definite need for treatment self-perception. According to the normative need DHC, $36.5 \%$ of the participants were borderline need to seek orthodontic treatment, $24.1 \%$ was in a definite need for treatment. Table 3 shows the distribution of the perceived treatment need by both young adults and the orthodontists. The kappa test $(\mathrm{kappa}=0.14, p<0.001)$ shows a certain level of agreement between young adults and orthodontists, even though the agreement is weak. A moderate agreement was observed between orthodontists perceived treatment need and normative need for treatment (kappa $=0.332, p<0.001)($ Table 4$)$. DHC was more critical than perceived judgments according to orthodontist's AC. There was a correlation between the components of IOTN, young adult's AC, orthodontist's $\mathrm{AC}$ and DHC (Table 5). A significant positive relationship $(p<0.001)$ between the young adult's AC and orthodontist's AC scores $(r=0.275)$; young adult's AC and the normative need DHC $(r=0.195)$, the orthodontist perception and the normative need $(r=0.743)$. Table 6 shows the correlations between the young adult's AC and gender $(r=0.143, p=0.007)$ and, EPQ-E $(r=-0.112$, $p=0.037)$.

\section{Discussion}

The aim of this study was to assess the agreement between orthodontists and the young adult's perception regarding to the IOTN-AC. We found that young adults tend to be less critical in assessing orthodontic treatment needs than orthodontists. However, the orthodontist's AC reflecting subjective treatment need is strongly connected to the normative need. Due to the complex doctor-patient relationship, orthodontists in China must consider this consistency [26].

This study focused on the agreement between orthodontists and young adult's perception of dental malocclusions. Although an agreement in perception was observed, the value indicated that it was not clinically relevant. Orthodontists perceived $36.2 \%$ of the young adults to be in need for treatment, but only $11 \%$ of the young adults were considered that need receive orthodontic treatment. The contrast exits because the present group presumably has less awareness of dental function

Table 3 Distribution of young adults perceived treatment need in relation to orthodontists perceived treatment need as evaluated by $A C$

\begin{tabular}{llllll}
\hline & & \multicolumn{3}{l}{ Orthodontist's AC } & Total \\
\cline { 2 - 4 } & & 1.00 & 2.00 & 3.00 & \\
\hline Young adult's AC & 1.00 & 213 & 86 & 14 & 313 \\
& 2.00 & 4 & 13 & 6 & 23 \\
Total & 3.00 & 5 & 6 & 1 & 12 \\
\hline
\end{tabular}

1 mean: little or no need for treatment; 2 mean: borderline need; 3 mean: definite need for treatment; Kappa $=0.14, p<0.001$ 
Table 4 Distribution of orthodontist's perceived treatment need in relation to normative treatment need

\begin{tabular}{llllll}
\hline & & \multicolumn{3}{l}{ Orthodontist's AC } & Total \\
\cline { 3 - 5 } & & 1.00 & 2.00 & 3.00 & \\
\hline DHC & 1.00 & 136 & 1 & 0 & 137 \\
& 2.00 & 81 & 46 & 0 & 127 \\
& 3.00 & 5 & 58 & 21 & 84 \\
Total & & 222 & 105 & 21 & 348 \\
\hline
\end{tabular}

1 mean: little or no need for treatment; 2 mean: borderline need; 3 mean:

definite need for treatment; Kappa $=0.332, p<0.001$

as well as dental aesthetics. Many studies have found that the motivation of the most people seeking orthodontic treatment is related to the appearance improvement and the desire for attractiveness [27, 28]. In the present study, the findings indicate that the opinion of the young adults does not align with the orthodontists. The young adults are not capable of understanding the severity of their presenting conditions. Therefore, better communication between orthodontists and young adults is necessary. Before providing treatment plans, it's better for orthodontists to explain to the young adults their dental conditions and why they have to take treatment into consideration. This communication helps to improve the patient's trust in their doctor in-charge and to obtain the compliance of the patient in the long-term orthodontic treatment duration.

It had been investigated that the choice for an orthodontic treatment demand were influenced by the desire for improvement in the appearance [3]. In this study, we try to explore the factors such as gender and personality traits that influence the subjective perception of aesthetic component among Chinese young adults. It is suggested that Chinese females are more critical with their dental appearance than the males. The result that females have a higher perception of aesthetic component is also supported by some other studies [29]. The study also shows that EPQ-E has some extent influence on subjective perception of aesthetic components. Young adults with higher introversion emotion are more critical with their dental aesthetic.

This study indicates that orthodontists should pay more attention to the perception discrepancy of aesthetics when young adults seek orthodontic treatment. Orthodontists should consider individual differences such as gender and

Table 5 Correlation between orthodontist and young adult's perception and normative need

\begin{tabular}{|c|c|c|c|}
\hline & & $\mathrm{DHC}$ & Orthodontist's AC \\
\hline \multirow{2}{*}{$\begin{array}{l}\text { Spearman's } \\
\text { correlation }\end{array}$} & young adult's AC & $0.195^{* *}$ & $0.275^{* *}$ \\
\hline & Orthodontist's AC & $0.743^{* *}$ & 1.000 \\
\hline
\end{tabular}

** $p<0.001,{ }^{*} p<0.05$
Table 6 Factors that correlate patient perception by IONT-AC

\begin{tabular}{llllll}
\hline & Gender & $\begin{array}{l}\text { T score } \\
\text { of } \mathrm{E}\end{array}$ & $\begin{array}{l}\text { T score } \\
\text { of } \mathrm{P}\end{array}$ & $\begin{array}{l}\text { T score } \\
\text { of } \mathrm{N}\end{array}$ \\
\hline $\begin{array}{l}\text { Spearman's } \\
\text { correlation }\end{array}$ & Young & $.143^{* *}$ & $-.112^{*}$ & 0.027 & 0.064 \\
& adult's & 0.007 & .037 & 0.621 & 0.235 \\
\hline
\end{tabular}

** $p<0.001,{ }^{*} p<0.05$

personality traits. Be aware of the personality traits associated with the patient's mental state and help the orthodontists communicate with the patient during the treatment [30]. The psychological status of the patient before any orthodontic treatment should not be overlooked, since it can be closely related to certain difficulties during the treatment, including the patient's discomfort and dissatisfaction [31, 32]. In the future, we will recruit samples from different cities to further explore and identify these findings in multi-center studies. In addition, the difference in dental aesthetic understanding between the patient and the orthodontist should be carefully considered in the patient's doctor's relationship. Also, we will try to investigate whether the psychological state will interfere with the outcome of orthodontic treatment.

\section{Conclusions}

Young adults tend to be less critical in assessing orthodontic treatment needs than orthodontists, and the orthodontist's AC reflecting subjective treatment need is strongly connected to the normative need. Furthermore, the adult's perception of aesthetic component is affected by gender and personality traits.

Abbreviations

AC: Aesthetic components; DHC: Dental Health Components; EPQ: Eysenck Personality Questionnaire; IOTN: the Index of Orthodontic Treatment Need

\section{Acknowledgements}

We are indebted to participants for providing an excellent study sample. And we appreciate the dentists who took clinical examination.

\section{Availability of data and materials}

The datasets used and analysed during the current study are available from the corresponding author on reasonable request.

\section{Authors' contributions}

LF conceived and designed the study, LF, CY and DWL performed the experiments, $C Y$ and YSJ collected data, DWL and YYL analyzed the data, CY wrote and revised the paper. All authors have read and approved the final version of the manuscript.

\section{Authors' information}

CY is a Resident physician at Department of Orthodontics, School of Stomatology, Wenzhou Medical University, Wenzhou, Zhejiang, P. R. China. DWL is a Undergraduate Student at School of Stomatology, Wenzhou Medical University, Wenzhou, Zhejiang, P. R. China.

LF is a Attending physician at Department of Orthodontics, School of Stomatology, Wenzhou Medical University, Wenzhou, Zhejiang, P. R. China. YSJ is a Undergraduate Student at School of Stomatology, Wenzhou Medical University, Wenzhou, Zhejiang, P. R. China.

YYL is a Undergraduate Student at School of Stomatology, Wenzhou Medical University, Wenzhou, Zhejiang, P. R. China. 


\section{Ethics approval and consent to participate}

All of the protocols were reviewed and approved by the Health Research Ethics Board of at Wenzhou Medical University. Written informed consent (including the release for dental records) was obtained from each participant.

\section{Competing interests}

The authors declare that they have no competing interests.

\section{Publisher's Note}

Springer Nature remains neutral with regard to jurisdictional claims in published maps and institutional affiliations.

\section{Author details}

'Department of Orthodontics, School and Hospital of Stomatology, Wenzhou Medical University, West Xueyuan Road 373, Wenzhou, Zhejiang 325000, People's Republic of China. ${ }^{2}$ School of Stomatology, Wenzhou Medical University, Wenzhou, Zhejiang, People's Republic of China. ${ }^{3}$ School and Hospital of Stomatology, Shandong University, Jinan, Shandong, People's Republic of China.

Received: 5 December 2017 Accepted: 6 June 2018 Published online: 19 June 2018

\section{References}

1. Xiao-Ting $L$, Tang $Y$, Huang $X L$, Wan $H$, Chen $Y X$. Factors influencing subjective orthodontic treatment need and culture-related differences among Chinese natives and foreign inhabitants. Int J Oral Sci. 2010; 2:149-57.

2. Pabari S, Moles DR, Cunningham SJ. Assessment of motivation and psychological characteristics of adult orthodontic patients. Am J Orthod Dentofac Orthop. 2011;140:263-72.

3. Chu $\mathrm{CH}$, Choy BH, Lo EC. Occlusion and orthodontic treatment demand among Chinese young adults in Hong Kong. Oral Health Prev Dent. 2009;7:83-91.

4. Dean JA, McDonald SM, Walker PO. Public assistance orthodontic treatment needs: a report from the state of Indiana. J Public Health Dent. 2005;65:133-7

5. Klages U, Bruckner A, Guld Y, Zentner A. Dental esthetics, orthodontic treatment, and oral-health attitudes in young adults. Am J Orthod Dentofac Orthop. 2005;128:442-9.

6. Albino JE, Lawrence SD, Tedesco LA. Psychological and social effects of orthodontic treatment. J Behav Med. 1994;17:81-98.

7. Klages U, Rost F, Wehrbein H, Zentner A. Perception of occlusion, psychological impact of dental esthetics, history of orthodontic treatment and their relation to oral health in naval recruits. Angle Orthod. 2007;77:675-80

8. Bellot-Arcis C, Montiel-Company JM, Pinho T, Almerich-Silla JM. Relationship between perception of malocclusion and the psychological impact of dental aesthetics in university young adults. J Clin Exp Dent. 2015;7:18-22.

9. Shue-Te Yeh M, Koochek AR, Vlaskalic V, Boyd R, Richmond S. The relationship of 2 professional occlusal indexes with patients' perceptions of aesthetics, function, speech, and orthodontic treatment need. Am J Orthod Dentofac Orthop. 2000;118:421-8.

10. Koochek AR, Yeh MS, Rolfe B, Richmond S. The relationship between index of complexity, outcome and need, and patients' perceptions ofmalocclusion: a study in general dental practice. Br Dent J. 2001;191:325-9.

11. Ousehal L, Lazrak L, Serrhini I, Elquars F. Evaluation of facial esthetics by a panel of professionals and a lay panel. Int Orthod. 2011:9:224-34.

12. Hamdan AM. The relationship between patient, parent and clinician perceived need and normative orthodontictreatment need. Eur J Orthod. 2004:26:265-71.

13. Grzywacz I. Orthodontic treatment needs and indications assessed with IONT. Ann Acad Med Stetin 2004:50:115-22

14. So LLY, Tang ELK. A comparative study using the occlusal index and the index of orthodontic treatment need. Angle Orthod. 1993:63:57-64.

15. Richmond S, Buchanan IB, Burden DJ, O'Brien KD, Andrews M, Roberts CT, Turbill EA. Calibration of dentists in the use of occlusal indices. Community Dent Oral Epidemiol. 1995;23:173-6.

16. Brook PH, Shaw WC. The development of an index of orthodontic treatment priority. Eur J Orthod. 1989;11:309-20.
17. Cardoso CF, Drummond AF, Lages EM, Pretti H, Ferreira EF, Abreu MH. The dental aesthetic index and dental health component of the index of orthodontic treatment need as tools in epidemiological studies. Int J Environ Res Public Health. 2011:8:3277-86.

18. Sharma J, Sharma RD. IOTN - a tool to prioritize treatment need in children and plan dental health services. Oral Health Dent Manag. 2014;13:65-70.

19. Borzabadi-Farahani A. An insight into four orthodontic treatment need indices. Prog Orthod. 2011;12:132-42.

20. Borzabadi-Farahani A. A review of the oral health-related evidence that supports the orthodontic treatment need indices. Prog Orthod. 2012; 13:314-25.

21. Gong Y. Use of the Eysenck personality questionnaire in China. Personal Individ Differ. 1984:5:431-8.

22. Liu XH. Eysenck personality questionnaire and Eysenck personality theory. Chin J Neurol Psychiatry. 1984;17:53-5.

23. Li X, Wang Q, Wu Y, Wang S, Huang Y, Li T. Personality characteristics and neurocognitive functions in parents of children with AutismSpectrum disorder. Shanghai Arch Psychiatry. 2017:29:41-7.

24. Borzabadi-Farahani A. A review of the evidence supporting the aesthetic orthodontic treatment need indices. Prog Orthod. 2012:13:304-13.

25. Viera AJ, Garrett JM. Understanding interobserver agreement: the kappa statistic. Fam Med. 2005:37:360-3.

26. Zhou $F$. The doctor can do first for the better patient-doctor relationship. Zhonghua Yi Xue Za Zhi. 2014:94:1371-2.

27. Mugonzibwa EA, Kuijpers-Jagtman AM, Van't Hof MA, Kikwilu EN. Perceptions of dental attractiveness and orthodontic treatment need among Tanzanian children. Am J Orthod Dentofac Orthop. 2004;125: 426-33.

28. Grzywacz I. The value of the aesthetic component of the index of orthodontic treatment need in the assessment of subjective orthodontic treatment need. Eur J Orthod. 2003:25(1):57-63.

29. Yin L, Chen WJ, Yu XZ, Yu J, Fang L, Zhou B, Li GY. A survey of perception differences of malocclusion between 16 to 22-year-old young adults and orthodontists. West China J Stomatol. 2011;29:153-6. 160

30. Phillips C, Bailey L, Kiyak HA, Bloomquist D. Effects of a computerized treatment simulation on patient expectations for orthognathic surgery. Int $J$ Adult Orthod Orthognathic Surg. 2001:16:87-98.

31. Kiyak HA, McNeill RW, West RA, Hohl T, Heaton PJ. Personality characteristics as predictors and sequelae of surgical and conventional orthodontics. Am J Orthod. 1986;89:383-92.

32. Neal CE, Kiyak HA. Patient perceptions of pain, paresthesia, and swelling after orthognathic surgery. Int J Adult Orthodon Orthognath Surg. 1991;6: 169-81

\section{Ready to submit your research? Choose BMC and benefit from:}

- fast, convenient online submission

- thorough peer review by experienced researchers in your field

- rapid publication on acceptance

- support for research data, including large and complex data types

- gold Open Access which fosters wider collaboration and increased citations

- maximum visibility for your research: over $100 \mathrm{M}$ website views per year

At BMC, research is always in progress.

Learn more biomedcentral.com/submissions 